\title{
Optical properties of metamaterial-based devices modulated by a liquid crystal
}

\author{
Filiz Karaomerlioglu • Amirullah M. Mamedov • \\ Ekmel Ozbay
}

Received: 12 May 2014/Accepted: 11 August 2014/Published online: 26 August 2014

(C) Springer-Verlag Berlin Heidelberg 2014

\begin{abstract}
Due to the fact that it is possible to manipulate light with photonic crystals (PCs), PCs hold a great potential for designing new optical devices. There has been an increase in research on tuning the optical properties of PCs to design devices. We presented a numerical study of optical properties of metamaterial-based devices by liquid crystal infiltration. The plane wave expansion method and finite-difference time-domain method for both TE and TM modes revealed optical properties in photonic crystal structures in an air background for a square lattice. E7 type has been used as a nematic liquid crystal and $\mathrm{SrTiO}_{3}$ as a ferroelectric material. We showed the possibility of the metamaterials for a two-dimensional photonic crystal cavity on a ferroelectric base infiltrated with a nematic liquid crystal.
\end{abstract}

\section{Introduction}

Progress in solid-state physics, optics of 3D artificial structures, and nanotechnologies based on a variety of the physical and chemical processes has strongly stimulated and motivated the investigation into the properties of

F. Karaomerlioglu ( $\square)$

Department of Electrical and Electronics Engineering, Mersin University, Mersin, Turkey

e-mail: filizkrm@gmail.com; filizkrm@mersin.edu.tr

F. Karaomerlioglu · A. M. Mamedov · E. Ozbay

Nanotechnology Research Center (NANOTAM),

Bilkent University, Ankara, Turkey

A. M. Mamedov

International Scientific Center, Baku State University,

Baku, Azerbaijan photonic crystals (PCs) and has resulted in the growth of applications of photonic band gap (PBG) materials, i.e., artificially structured materials where the optical parameters are periodically modulated in space with a period of a unit PC cell on the order of the optical wavelength. Previous studies about PBG structures, PBG materials, and PCs have been important investigations [1, 2]. The basic feature of PCs is the presence of permitted and forbidden frequency bands for light. It is possible to manipulate the light with PCs. Due to this property, PCs hold great potential for designing new optical devices. There has been an increase in research on tuning the optical properties of PBG to design devices. Some tunable PBG research has been conducted in one-dimensional (1D), two-dimensional (2D), and three-dimensional (3D) [3-6] PCs.

Recently, new investigations have reached a point of view of LCs because of the tunable light wave propagation. LCs' refractive indices can be changed by rotating the directors of LCs [7]. Liu investigated the tunable light wave propagation in 2D hole-type PCs infiltrated with nematic liquid crystal and the tunable absolute band gap in 2D anisotropic photonic crystal structures modulated by a nematic liquid crystal [8, 9]. Liu and Chen proposed the tunable field-sensitive polarizer using hybrid conventional waveguides and PC structures with nematic liquid crystals, the tunable PC waveguide coupler with nematic liquid crystals, the tunable PC waveguide Mach-Zehnder interferometer based on nematic liquid crystals, the tunable full band gap in a 3D PC structure modulated by a nematic liquid crystal, the tunable channel drop filter in a $2 \mathrm{D}$ photonic crystal modulated by a nematic liquid crystal, and the tunable band gap in a photonic crystal modulated by a nematic liquid crystal [10-15]. Liu et al. [16, 17] created the tunable band gap in 3D anisotropic photonic crystal structures modulated by a nematic liquid crystal and an efficient 
tunable negative refraction photonic crystal achieved by an elliptic rod lattice with a nematic liquid crystal.

Negative refraction has been investigated recently. In contrast to double-negative metamaterials (left-handed materials), single-negative materials, and indefinite materials, PCs made of synthetic periodic dielectric materials can exhibit negative refraction behaviors that are solely determined by the characteristics of their band structures and equi-frequency contours [18-21].

In the present paper, we theoretically demonstrated and developed the optical properties in the 2D PC structure of ferroelectric rods modulated by nematic LCs. The investigation was achieved by controlling the intensity of the optical properties that had different materials added to a certain structure.

\section{Method of calculation}

The fundamentals of the plane wave expansion (PWE) method and finite-difference time-domain (FDTD) method are based on a direct numerical solution of the timedependent Maxwell's equations as illustrated [22].

On the other hand, Bloch's theorem [23] is used to expand the $H(\vec{r})$ field in terms of plane waves since the light waves are transmitted in periodic structures, as

$H(\vec{r})=\sum_{G} h(\vec{G}) \hat{e}_{\vec{G}} \mathrm{e}^{i(\vec{k}+\vec{G}) \cdot \vec{r}}$

where $\vec{k}$ is a wave vector in the Brillouin zone of the lattice and $\hat{e}_{\vec{G}}$ is the direction that is perpendicular to the wave vector $(\vec{k}+\vec{G})$ owing to the transverse character of the magnetic field $H(\vec{r}), \nabla \cdot H(\vec{r})=0$.

\section{Results of the calculation}

The PBG is manipulated by the rotating directors of LCs under the impact of an applied electric field. Using the PWE and the FDTD methods, the PC structure, composed of a PC in ferroelectric rods infiltrated with nematic LCs in an air background, is designed for the square lattice. PCs structures that are designed as round rods and square rods with a square lattice shape are computed. $\mathrm{SrTiO}_{3}$ was used as ferroelectric material and E7 type as nematic LCs.

This paper is aimed at describing and comparing 2D PC structures which differ by the characteristics of their band gap, transmission, equi-frequency, and group velocity dependences.

\subsection{Band gap and transmission}

We considered the results obtained from the calculation of the band structure of the spectrum for the sample of the $2 \mathrm{D}$ $\mathrm{PC}$ of the $\mathrm{SrTiO}_{3}$ rods type, which consists of the elements in the form of dielectric cylinder forming a square lattice filled without and with LC. The calculations are performed for the PCs with the permittivity of the cylinders 5.772 and the period of the structure $a=1 \mu \mathrm{m}$. E7 type LCs have two different principle refractive indices as the ordinaryrefractive index $n_{0}=1.52131$ and the extraordinary refractive index $n_{\mathrm{e}}=1.73657$. The filling factor is $30 \%$.

Schematic views of the proposed 2D PC of $\mathrm{SrTiO}_{3}$ round and square rods with nematic LC-infilled in an air background $\left(\varepsilon_{\mathrm{a}}=1\right)$ in a square lattice are shown in Fig. 1a. The photonic band structure for TE and TM mode is calculated along direction that includes the high symmetry points $\Gamma, X$ and $M$ for the Brillouin zone in a square lattice. It is assumed that $r_{1}=0.3 a$ and $r_{3}=0.1 a$ denote the outer radius and inner radius of $\mathrm{SrTiO}_{3}$ round rods, and $r_{2}=0.2 a$ round rods air-infilled.

There is no band gap for 2D PC of $\mathrm{SrTiO}_{3}$ round and square rods with air-infilled and rods with nematic LCinfilled in TE mode.

Figure 2 shows the seed band structure of the PC (inset, from Fig. 1a) with the permittivity of the dielectric cylinders and the permittivity of free space 1. For the filling factor $30 \%$, this $\mathrm{PC}$ has the maximum width of the first complete band gap. In this case, the PC band gap along the direction between high symmetry points $\Gamma-X$ direction of the Brillouin zone lies in the frequency range from
Fig. $12 \mathrm{D}$ PC structure for square lattice. a $\mathrm{SrTiO}_{3}$ round rods, $\mathbf{b ~} \mathrm{SrTiO}_{3}$ square rods in an air background (a)

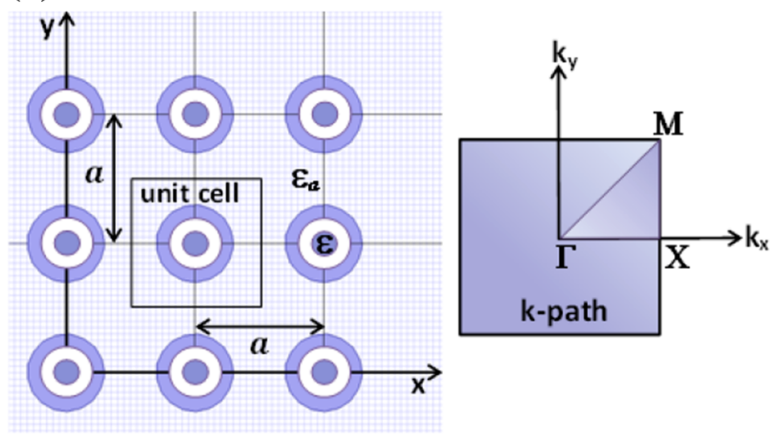

(b)

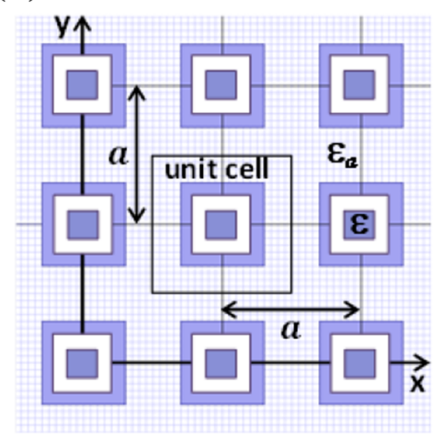


$1.049(2 \pi c / a)$ to $1.092(2 \pi c / a)$. For $\mathrm{SrTiO}_{3}$ round rods with air-infilled, relative widths are $4.07 \%$ of TM mode.

The dispersion of the PC structure in combination with the dispersion of the LC leads to the appearance of additional band gaps in the continuous spectrum of the seed PC and additional narrow transmission bands in the band gap of the PC (which are imperceptible on the scale of Fig. 2). These effects are illustrated in Fig. 3. It can be seen from Fig. 3 that the presented fragment of the band structure of the spectrum exhibits an additional band gap with the width $\Delta \omega=\omega-\omega_{0}$. This width exceeds the width of the LC resonance lines by an order of magnitude when the frequencies of the LC $1.024(2 \pi c / a)$ and $1.063(2 \pi c / a)$ are in the continuous spectrum near the high-frequency edge of the third band gap.

A different situation arises when the LC frequencies $0.365(2 \pi c / a), 0.623(2 \pi c / a)$, and $1.024(2 \pi c / a)$ are in the band gap of the PC (Fig. 3). In this case, a narrow transmission band with the width exceeding the width of the LC lines by an order of magnitude appears in the band gap.

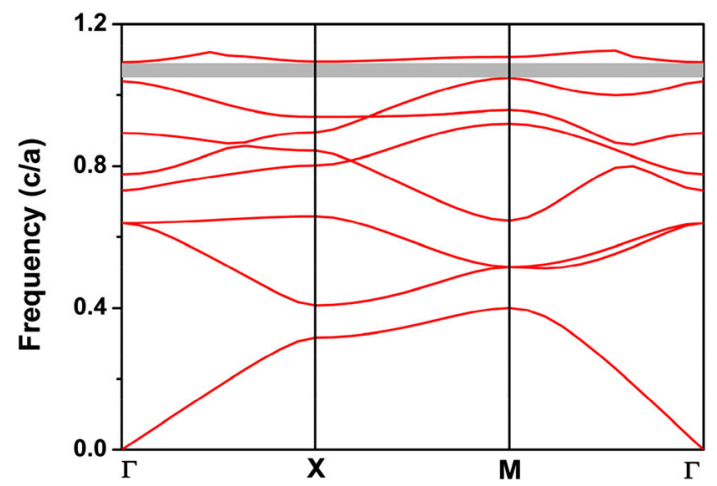

Fig. 2 The photonic band structure of TM mode in square lattice for $\mathrm{SrTiO}_{3}$ round rods with air-infilled

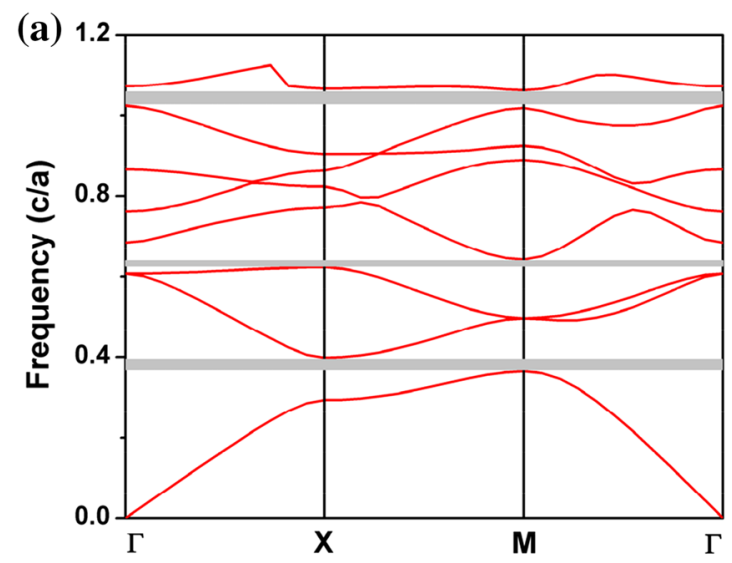

The widths of the additional transmission band and the band gap can be controlled by varying the type of nematic LC.

When $\mathrm{SrTiO}_{3}$ round rods are infiltrated with nematic LC, three band gap in TM mode for the extraordinary refractive index is shown in Fig. 3a. The band gap has relative widths of $8.68,2.98$, and $3.72 \%$, and center normalized frequencies of $0.382(2 \pi c / a), 0.633(2 \pi c / a)$, and $1.044(2 \pi c / a)$. As the results obtained for ordinary-refractive index and extraordinary refractive index of nematic LC are different, it is an indication of anisotropy. Using the anisotropic features of LCs, different results for TE and TM mode were obtained. This means that either the structure is rotated into a certain direction or it is infiltrated with-LCs - that have different directors (orientations of the LCs molecules along order's direction) into the structure in order to be able to change the features of these structures.

It is well known that an anisotropic nanostructuring photonic band structure array is capable of changing the polarization state of transmitted or reflected light. Therefore, we also calculated the optical response of the photonic band structure for different directors of the LCs and the light polarization transmitted through a photonic band array. The numerical results of the variation of full transmission by changing the director of LC for the PC structure are presented. The transmission spectrum as a function of the frequency is computed for extraordinary refractive index of nematic LC in a square lattice. The transmission of $\mathrm{SrTiO}_{3}$ round rods with nematic LC-infiltrated is zero at frequencies between $0.296(2 \pi c / a)$ and $0.394(2 \pi c / a)$, between $0.614(2 \pi c / a)$ and $0.679(2 \pi c / a)$, between $0.863(2 \pi c / a)$ and $0.891(2 \pi c / a)$, between $1.021(2 \pi c / a)$ and $1.054(2 \pi c / a)$, and between $1.089(2 \pi c / a)$ and $1.194(2 \pi c / a)$ (Fig. 3b).

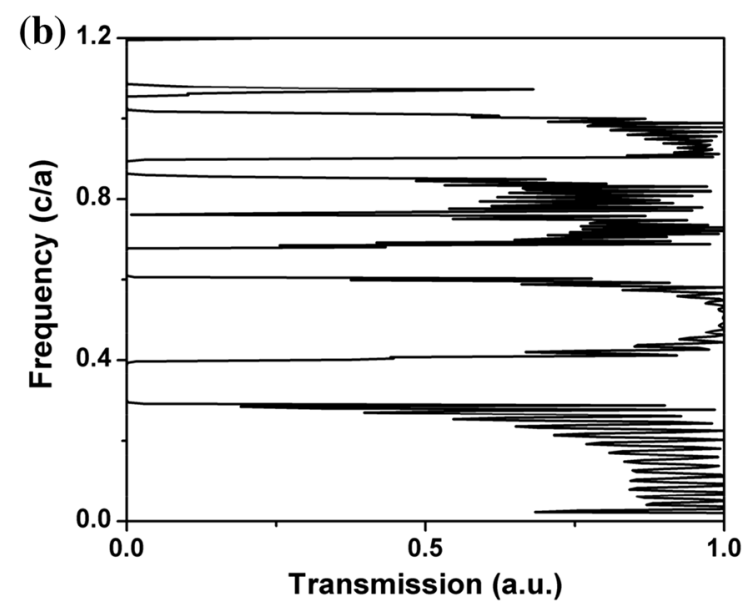

Fig. 3 a The photonic band structure in TM mode b; transmission spectrum of $\mathrm{SrTiO}_{3}$ round rods with nematic LC-infilled with extraordinary refractive index in air background in square lattice 


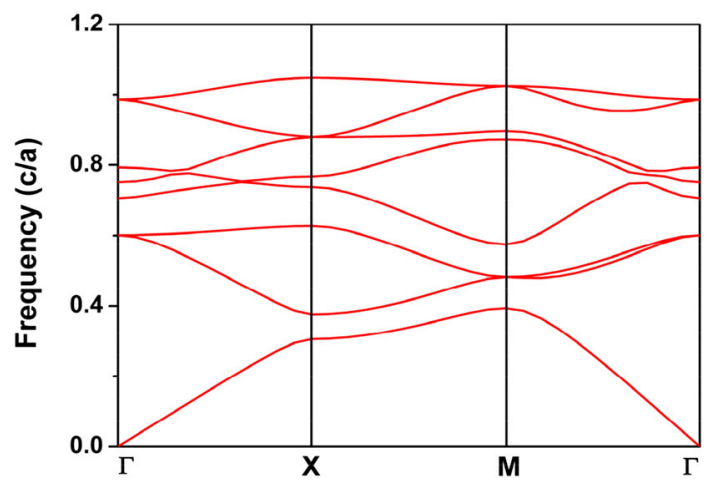

Fig. 4 The photonic band structure in TM mode of $\mathrm{SrTiO}_{3}$ square rods with air-infilled in air background in square lattice

Let us turn to the analysis of the results obtained from the calculation of the band structure of the spectrum for the sample of a PC infiltrated by $\mathrm{LC}$ of the $\mathrm{SrTiO}_{3}$ round rods type, which consists of elements in the form of infinite hollow cylinders that are filled with an LC and form square lattice in a dielectric matrix. Figure 2 shows the seed band structure for the $\mathrm{SrTiO}_{3}$ round rods type PCs sample with the parameters the permittivity of the air 1 , and the filling factor $30 \%$.

For the filling factor $30 \%$, this $\mathrm{PC}$ has the maximum width of the complete band gap. In this case, the photonic band gap along the direction between high symmetry points $\Gamma-X$ direction of the Brillouin Zone lies in the frequency range around $1.07(2 \pi c / a)$.

A comparison of Figs. 2 and 3 shows that the spectra of seed PC of both types have a similar structure. This is obviously explained by the fact that these crystals have close factors of filling with dielectric materials.

Structure and material are very important to determine the optical properties of a PC structure as aforementioned. Therefore, we changed the PC structure in order to obtain optimum results. The materials' refractive index values have the same values as in the above calculations.

We consider that 2D PC of $\mathrm{SrTiO}_{3}$ square rods in an air background in a square lattice with lattice constant $a=1 \mu \mathrm{m}$. Parameters $l_{1}=0.6 a$ and $l_{3}=0.2 a$ denote the outer and inner length of $\mathrm{SrTiO}_{3}$ and $l_{2}=0.4 a$ the length of the air, and the sides of the square rods are parallel to the primitive reciprocal lattice vectors in Fig. 1b. The photonic band structure is calculated along with the high symmetry point for the Brillouin zone in a square lattice (Fig. 4). According to Fig. 4, there is also no band gap in TM mode. When $\mathrm{SrTiO}_{3}$ square rods are compared with $\mathrm{SrTiO}_{3}$ round rods in a square lattice, it is seen that there is a reduction in the band gap number of the square structure.

The photonic band structure of $\mathrm{SrTiO}_{3}$ square rods modulated by nematic LC is studied again for extraordinary refractive index of LC. There is only one band gap in
TM mode as shown in Fig. 5a. Their relative widths are $2.63 \%$, and the center normalized frequencies are $0.361(2 \pi c / a)$.

The transmission spectrum of $\mathrm{SrTiO}_{3}$ square rods with nematic LC-infiltrated is zero at frequencies between $0.293(2 \pi c / a)$ and $0.383(2 \pi c / a)$, between $0.599(2 \pi c / a)$ and $0.664(2 \pi c / a)$, between $0.762(2 \pi c / a)$ and $0.776(2 \pi c / a)$, between $0.870(2 \pi c / a)$ and $0.891(2 \pi c / a)$, and between $1.075(2 \pi c / a)$ and $1.187(2 \pi c / a)$ in Fig. $5 b$. When the PC structure is turned into $\mathrm{SrTiO}_{3}$ square rods, it can be clearly seen that PBG has become narrow and that there was a decrease in the band gap.

\subsection{Equi-frequency surface}

Because the momentum conservation law is satisfied at the reflection and refraction of waves, it is convenient to analyze the reflection and refraction of a certain wave in the space of wave numbers $\sum_{\vec{k}}=\left\{0 ; k_{x}, k_{y}, k_{z}\right\}$ by introducing the equi-frequency surface of the wave. This surface is directly described by the dispersion relation of the anisotropic medium at the fixed frequency $\omega$. Then, the group velocity $\vec{V}_{\mathrm{g}}$ of the wave in an anisotropic medium can be found as the frequency gradient in the space of wave vectors [24]. It is known that for an electromagnetic wave propagating in an anisotropic medium with a fixed frequency $\omega$, the equi-frequency surface represents a sphere. In this case, the wave vector $\vec{k}$ and group velocity vector $\vec{V}_{\mathrm{g}}$, which determines the ray direction, are always parallel. However, equi-frequency surface is not spherical for anisotropic media, and the vectors $\vec{V}_{\mathrm{g}}$ and $\vec{k}$ are not parallel. By analogy with 3D case, the propagation, reflection, and refraction of the wave in 2D structure can be described in terms of the equi-frequency dependence which can be considered as the section of the dispersion surface $\omega\left(k_{y}, k_{z}\right)$ in the space of variables $\left\{\omega, k_{y}, k_{z}\right\}$ by the plane corresponding to constant frequency. It is well known that the analysis of equi-frequency dependences is most efficient in the studies of 2D geometries, especially in solving problems when only orientations of the $\vec{V}_{\mathrm{g}}$ and $\vec{k}$ vector of incident, reflected, and refracted waves are of interest and are not the amplitudes of the reflected and refracted rays. The equi-frequency dependence has a simple physical meaning for the analysis of 2D geometries: Since this dependence describe all the possible waves with the given frequency $\omega$ and various wave vectors, the directions of the reflected and the refracted rays can be determined by simply finding the points in equi-frequency dependences of media that satisfy the momentum conservation law at a known orientation of the boundary and a given angle of incidence of the wave. 

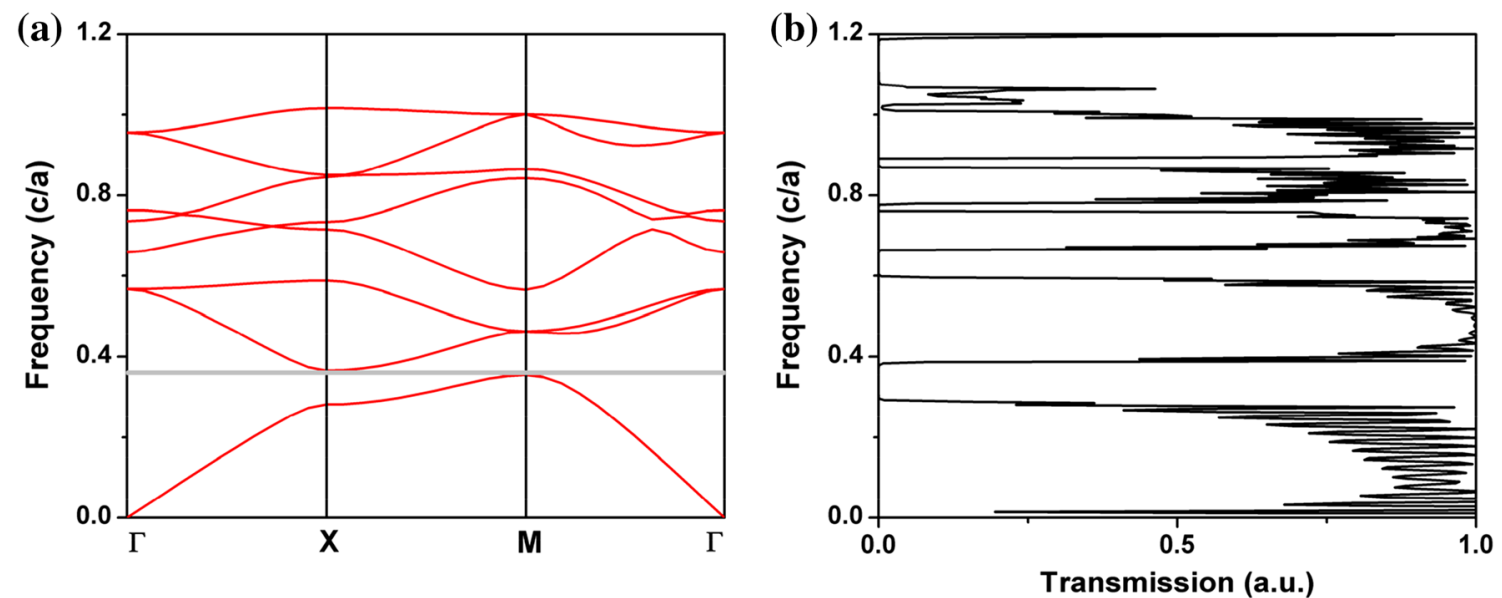

Fig. 5 a The photonic band structure in TM mode $\mathbf{b}$; transmission spectrum of $\mathrm{SrTiO}_{3}$ square rods with nematic LC-infilled with extraordinary refractive index in air background in square lattice
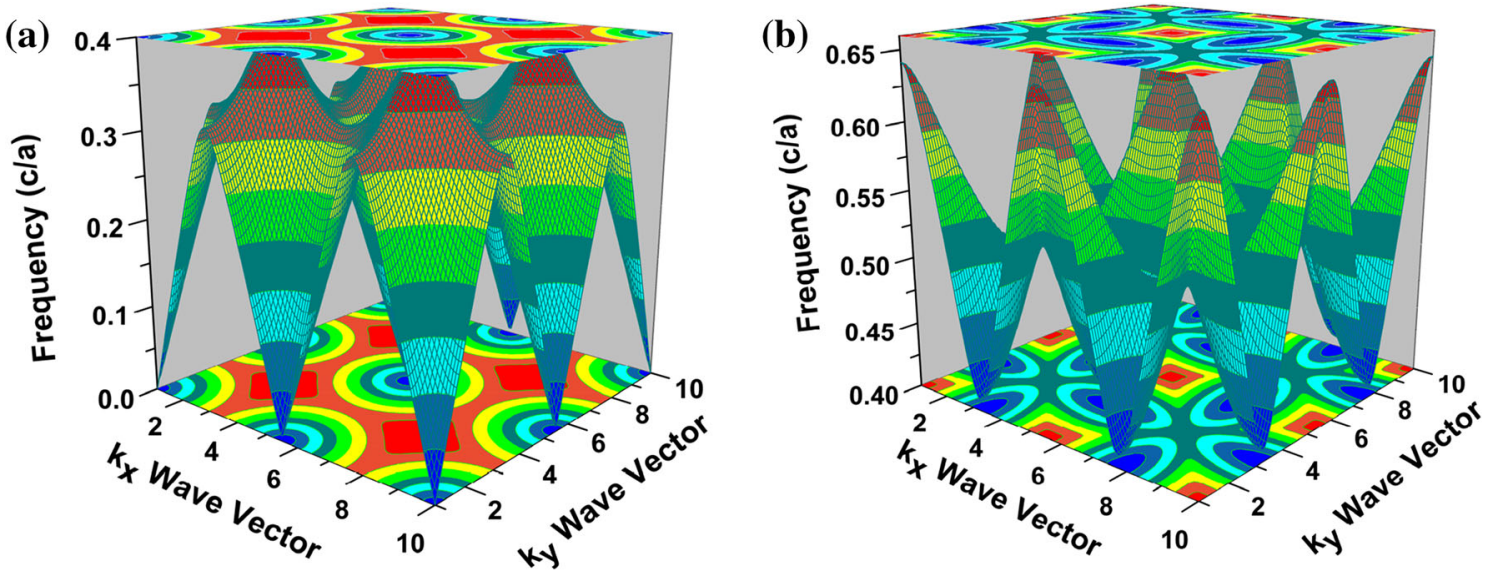

Fig. 6 The equi-frequency contours of $\mathrm{PC}$ with $\mathrm{SrTiO}_{3}$ round rods in an air background for the square lattice. a First band, b second band

Now, we present some numerical examples for our PC structures. In all these examples, we exploit symmetry to calculate the equi-frequency surfaces over the irreducible Brillouin Zone of the entire Brillouin Zone. First, we consider the equi-frequency surface of a square lattice of air hole in a dielectric medium for the $\vec{H}$-polarization. In Fig. 6, we reproduce PC with $\mathrm{SrTiO}_{3}$ round rods in an air background $\left(\varepsilon_{\mathrm{a}}=1\right)$ in the square lattice parameters the lattice constant $a=1 \mu \mathrm{m}$, radius $r_{1}=0.3 a$ and $r_{3}$ $=0.1 a$ and refractive index of $\mathrm{SrTiO}_{3} n=2.40252$, radius $r_{2}=0.2 a$ and refractive index of the air $n=1$. Here, the map was discretized using six field points per edge of the unit cell for the first band in Fig. 9a. The map was discretized using four field points per edge of the unit cell for the second band in Fig. 9b. The curves shown correspond to equi-frequency surfaces of the lowest order band up to frequencies just below the band gap starting at around $1.048(2 \pi c / a)$.
In Fig. 7, we reproduce for $\mathrm{PC}$ with $\mathrm{SrTiO}_{3}$ round rods of nematic LC-infill in an air background for the square lattice parameters the same for $\mathrm{SrTiO}_{3}$ round rods and extraordinary refractive index of E7 type LCs $n_{\mathrm{e}}=1.73657$ for radius $r_{2}=0.2 a$. The map was discretized using five field points per edge of the unit cell for the first band in Fig. 7a. For the second band, the map was discretized using four field points per edge of the unit cell in Fig. 7b. The curves shown correspond to equi-frequency surfaces of the lowest order band up to frequencies just below the band gap starting at around $0.365(2 \pi c / a)$. Because of the three band gap, the band gap from band 3 to band 4 is between $0.623(2 \pi c / a)$ and $0.642(2 \pi c / a)$, and the band gap from band 7 to band 8 is between $1.024(2 \pi c / a)$ and $1.063(2 \pi c / a)$.

In Fig. 8, we reproduce for $\mathrm{PC}$ with $\mathrm{SrTiO}_{3}$ square rods in an air background for the square lattice parameters $l_{1}=$ $0.6 a$ and $l_{3}=0.2 a$ the outer and inner length of $\mathrm{SrTiO}_{3}$ and 

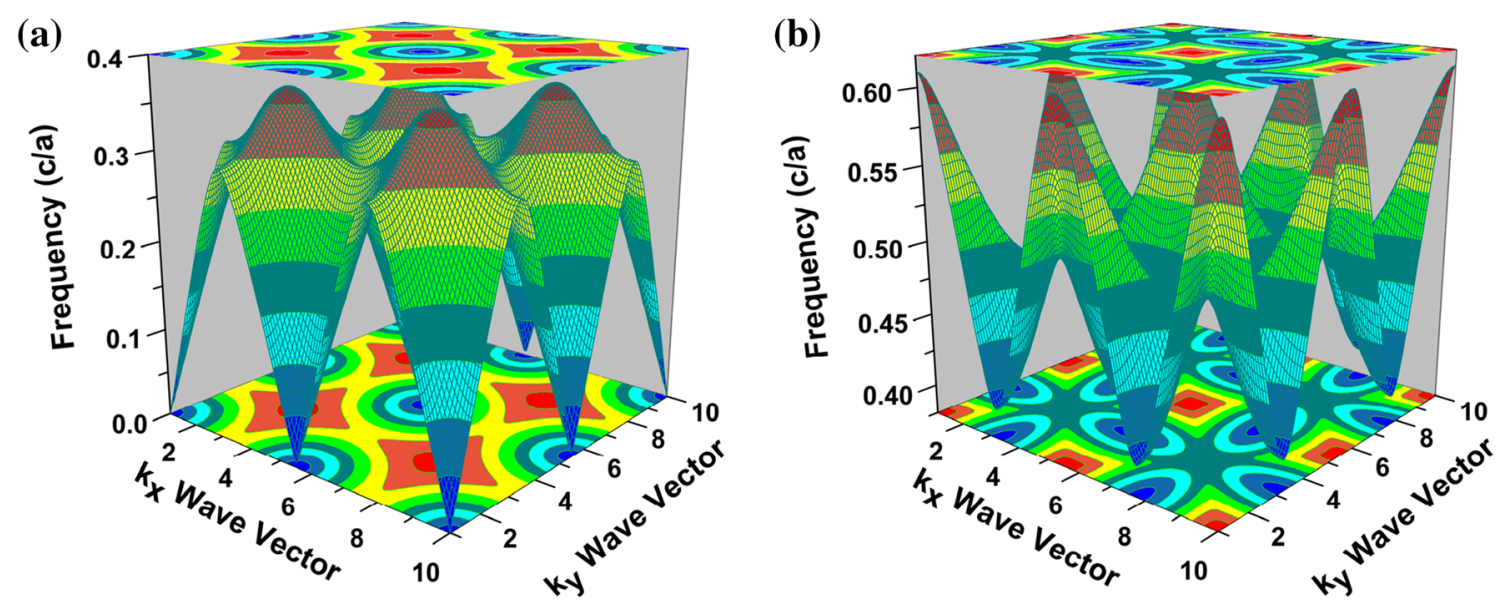

Fig. 7 The equi-frequency contours of PC with $\mathrm{SrTiO}_{3}$ round rods of nematic LC-infill in an air background for the square lattice. a First band, b second band
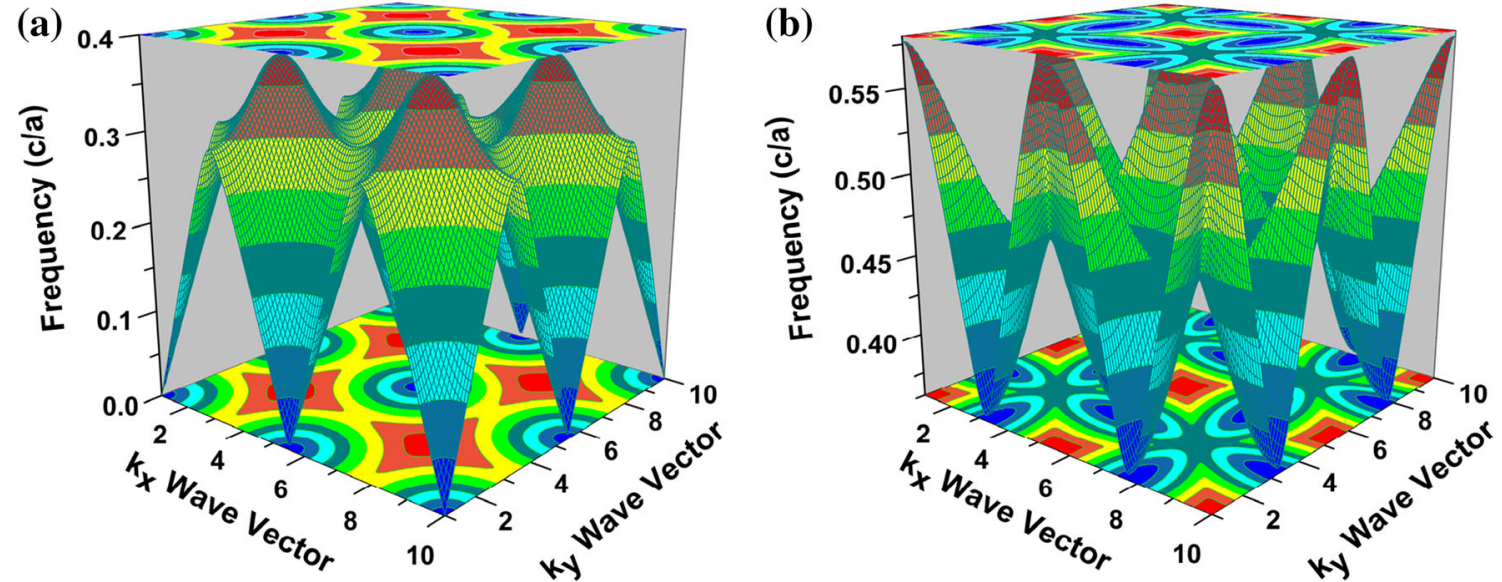

Fig. 8 The equi-frequency contours of $\mathrm{PC}$ with $\mathrm{SrTiO}_{3}$ square rods in an air background for the square lattice. a First band, b second band

$l_{2}=0.4 a$ the length of the air. For the first band, the map was discretized using five field points per edge of the unit cell (Fig. 8a). The map was discretized using four field points per edge of the unit cell for the second band in Fig. $8 \mathrm{~b}$. The curves shown correspond to equi-frequency surfaces of the non-band gap.

In Fig. 9, we reproduce for PC with $\mathrm{SrTiO}_{3}$ square rods of nematic LC-infill in an air background for the square lattice parameters the same for $\mathrm{SrTiO}_{3}$ square rods and $l_{2}=0.4 a$ the length of the LCs. The map was discretized using six field points per edge of the unit cell for the first band in Fig. 6a. For the second band, the map was discretized using four field points per edge of the unit cell (Fig. 6b). The curves shown correspond to the equifrequency surfaces of the lowest order band up to frequencies just below the band gap starting at around $0.356(2 \pi c / a)$.

\subsection{Group velocity}

We believe a pulse of light propagating along the PC and let the pulse be finite in dimension along the direction of propagation. Theoretically, such a pulse can be represented as a wave packet formed as a superposition of the modes, but with a different propagation constant [25]. From [25], it follows that the envelope of the wave packet propagates with the velocity $V_{\mathrm{g}}^{\mathrm{z}}=\omega_{n}(\beta)$, where $\beta$ is propagation constant. The direct calculation of the derivative of the dispersion relation calculated numerically is not always convenient and can give error. As shown in [26], the group velocity of the wave packet $V_{\mathrm{g}}^{\mathrm{z}}$, is equal to the velocity of energy transfer by the mode $n \beta$. Thus, by using the results of [26], the group velocity can be always calculated more accuracy, irrespective of the number of points in the dispersion curve. 

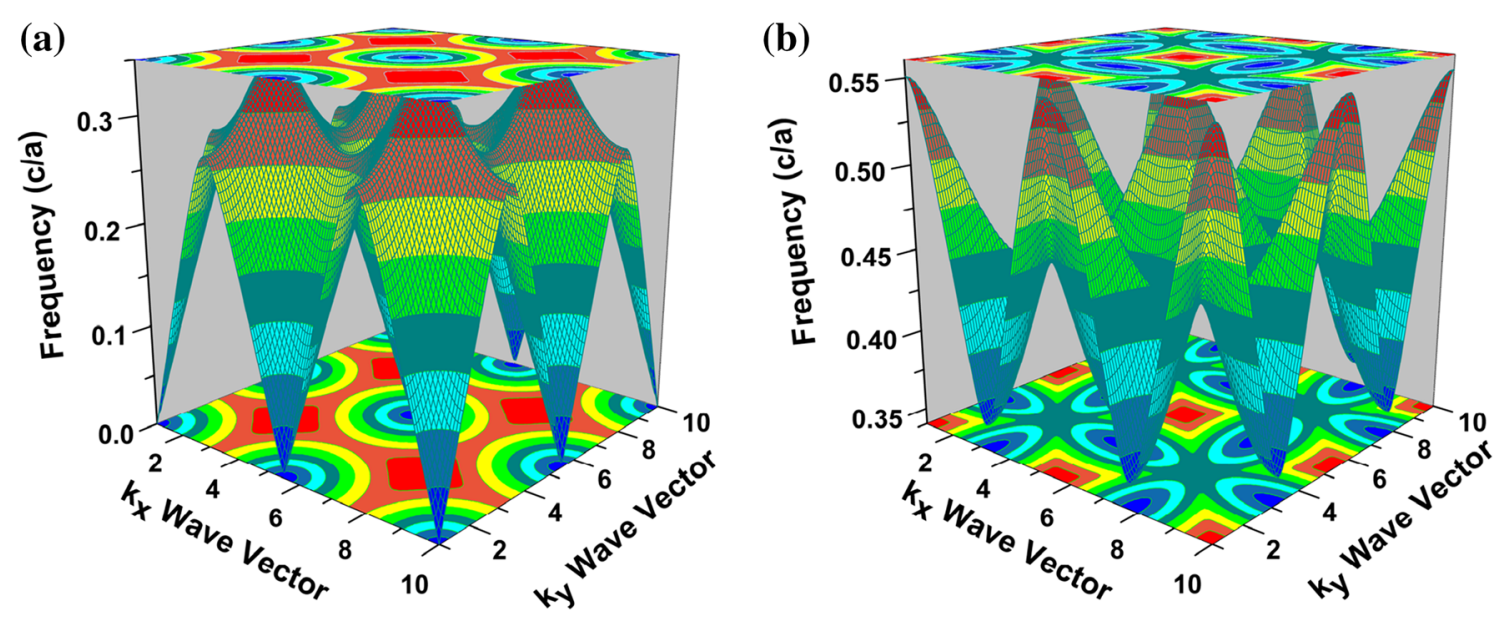

Fig. 9 The equi-frequency contours of $\mathrm{PC}$ with $\mathrm{SrTiO}_{3}$ square rods of nematic LC-infill in an air background for the square lattice. a First band, b second band
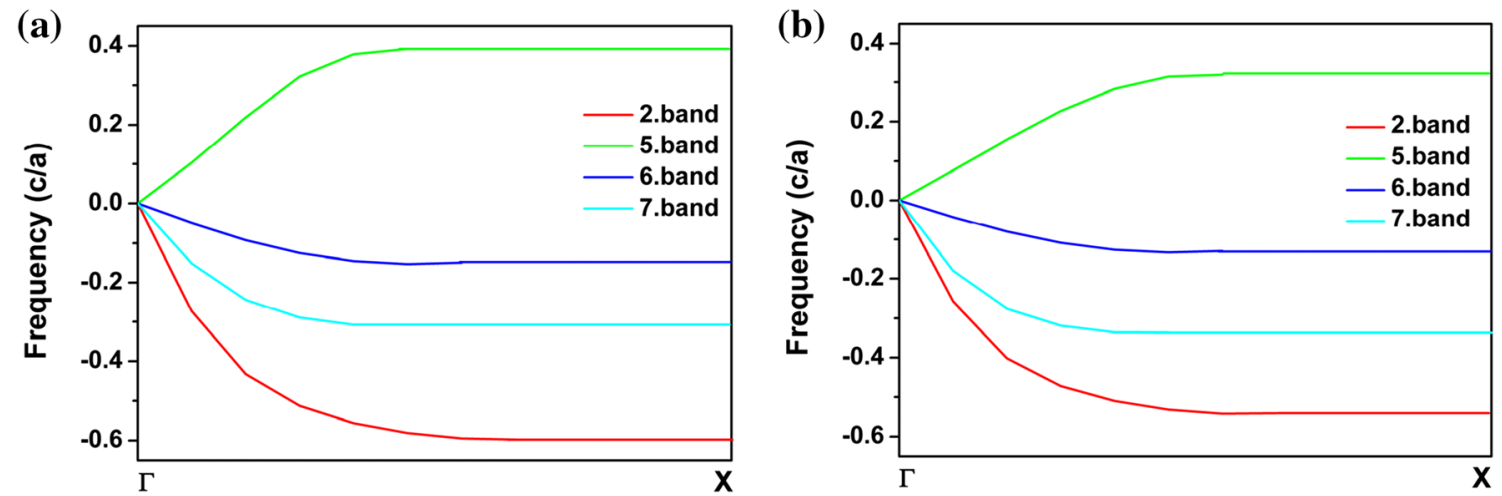

Fig. 10 The group velocity in $\mathrm{TM}$ mode of a $\mathrm{PC}$ with $\mathrm{SrTiO}_{3}$ round rods, $\mathbf{b} \mathrm{PC}$ with $\mathrm{SrTiO}_{3}$ round rods of nematic $\mathrm{LC}$-infill in an air background for the square lattice
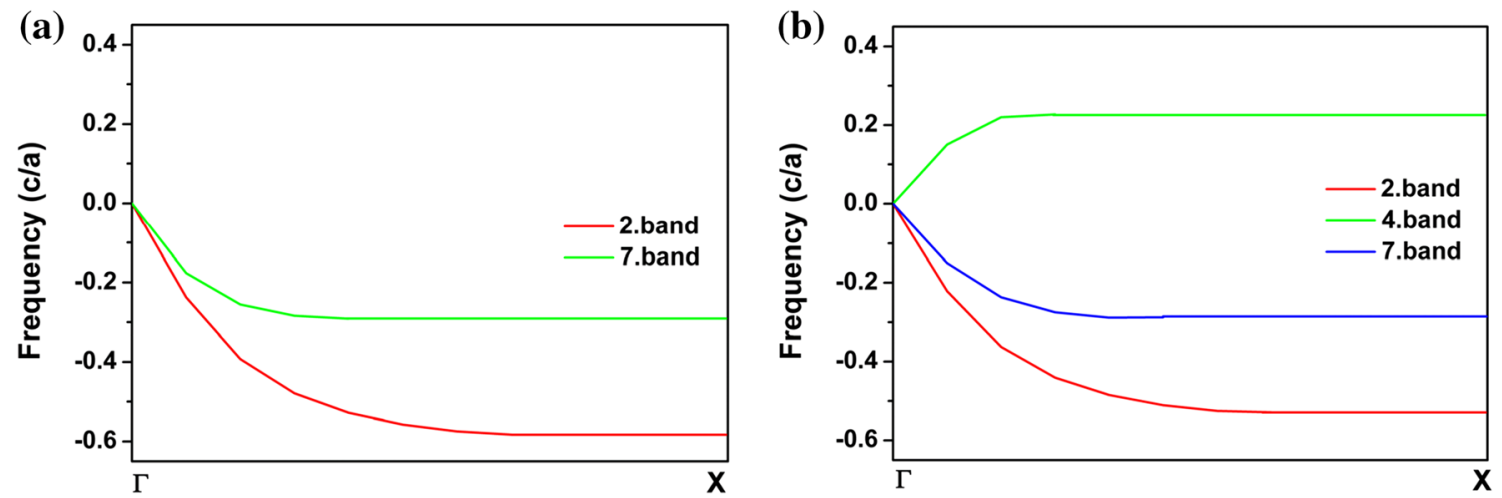

Fig. 11 The group velocity in $\mathrm{TM}$ mode of a PC with $\mathrm{SrTiO}_{3}$ square rods, b $\mathrm{PC}$ with $\mathrm{SrTiO}_{3}$ square rods of nematic LC-infill in an air background for the square lattice

Figures 10 and 11 show the results of calculations of the group velocity of the wave packet formed of the differently polarized localized modes in $\mathrm{PC}$ with $\mathrm{SrTiO}_{3}$ round rods, $\mathrm{PC}$ with $\mathrm{SrTiO}_{3}$ round rods of nematic LC-infill, $\mathrm{PC}$ with $\mathrm{SrTiO}_{3}$ square rods, and $\mathrm{PC}$ with $\mathrm{SrTiO}_{3}$ square rods of nematic LCinfill in an air background in the square lattice, respectively.

From Figs. 10 and 11, it is evident that the components of the group velocity versus the high symmetry direction 

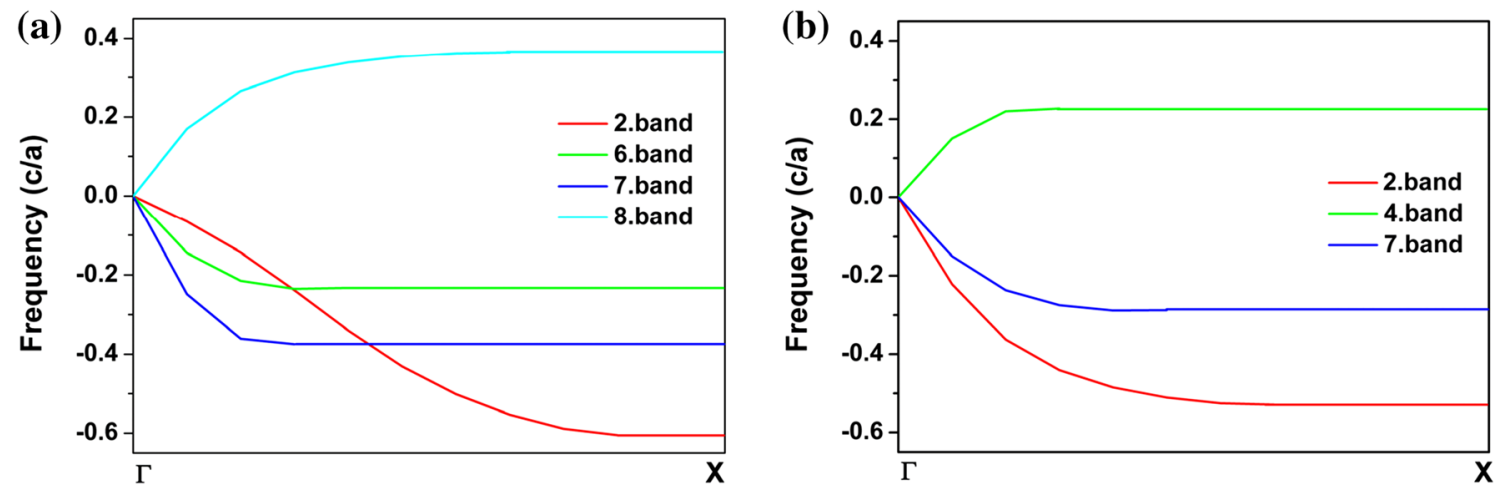

Fig. 12 The group velocity of $\mathrm{PC}$ with $\mathrm{SrTiO}_{3}$ square rods of nematic LC-infill in an air background for the square lattice in a TE mode, b TM mode

$(\Gamma-X)$ vary over wide limits. The dependences $V_{\mathrm{g}}^{\mathrm{z}}(\beta)$, that describe the wave packets of localized modes of any polarization and any order exhibit (generally) a maximum at certain propagation constants. This means that the dispersion of group velocity can be positive, negative, or zero [27].

Therefore, as can be seen in Fig. 12, the group velocity of TE-polarized wave packets is almost always higher than that of the TM-polarized packets.

\section{Conclusions}

We analyzed the optical properties in a 2D PC structure of ferroelectric round and square rods filled without and with nematic LCs in a square lattice. The photonic band structure for TE and TM mode is calculated along with the high symmetry point for the Brillouin zone.

It has been shown that the dispersion of $2 \mathrm{D}$ seed photonic structure in combination with the dispersion of nematic LC leads to qualitative changes in the band structure of the spectrum of the intrinsic electromagnetic excitations of the LC-infiltrated PC.

In practical applications, such LC-infiltrated PCs are promising materials for use in the design of narrow band filters and a new type of optoelectronic devices.

Acknowledgments One of the authors (Filiz Karaomerlioglu) would like to thank the Scientific and Technological Research Council of Turkey (TUBITAK) Postdoctoral Fellowship.

\section{References}

1. E. Yablonovitch, Photonic band-gap structures. J. Opt. Soc. Am. B 10, 283-295 (1993)

2. J.D. Joannopoulos, S.G. Johnson, J.N. Winn, R.D. Meade, Photonic Crystals: Molding the Flow of Light (Princeton University Press, Princeton, New Jersey, 2008)
3. C. Sibilia, T.M. Benson, M. Marciniak, T. Szoplik, Photonic Crystals: Physics and Technology (Springer, Milano, Italia, 2008)

4. K. Sakoda, Optical Properties of Photonic Crystals (Springer, Berlin, Germany, 2005)

5. M.C. Gupta, J. Ballato, The Handbook of Photonics (CRC Press, Boca Raton, New York, USA, 2007)

6. J.M. Brosi, Slow-Light Photonic Crystal Devices for High-Speed Optical Signal Processing (University of Karlsruhe, Karlsruhe, Germany, 2009)

7. I.C. Khoo, S.T. Wu, Optics and Nonlinear Optics of Liquid Crystals: Electro-Optical Properties of Liquid Crystals (World Scientific, Singapore, 1993), pp. 100-258

8. C.Y. Liu, Tunable lightwave propagation in two-dimensional hole-type photonic crystals infiltrated with nematic liquid crystal. Phys. E 44, 313-316 (2011)

9. C.Y. Liu, Creation of tunable absolute band gaps in a twodimensional anisotropic photonic crystal modulated by a nematic liquid crystal. Phys. Lett. A 372, 5198-5202 (2008)

10. C.Y. Liu, L.W. Chen, Tunable field-sensitive polarizer using hybrid conventional waveguides and photonic crystal structures with nematic liquid crystals. Opt. Commun. 256, 114-122 (2005)

11. C.Y. Liu, L.W. Chen, Tunable photonic-crystal waveguide Mach-Zehnder interferometer achieved by nematic liquid-crystal phase modulation. Opt. Express 12, 2616-2624 (2004)

12. C.Y. Liu, L.W. Chen, Tunable photonic crystal waveguide coupler with nematic liquid crystals. IEEE Photonics Technol. Lett. 16, 1849-1851 (2004)

13. C.Y. Liu, L.W. Chen, Tunable full band gap in a three-dimensional photonic crystal modulated by a nematic liquid crystal. Phys. E 35, 173-177 (2006)

14. C.Y. Liu, L.W. Chen, Tunable channel drop filter in a twodimensional photonic crystal modulated by a nematic liquid crystal. J. Nanomater. 1-6, 2006 (2006)

15. C.Y. Liu, L.W. Chen, Tunable band gap in a photonic crystal modulated by a nematic liquid crystal. Phys. Rev. B 72, 045133-045138 (2005)

16. C.Y. Liu, Y.T. Peng, J.Z. Wang, L.W. Chen, Creation of tunable band gaps in a three-dimensional anisotropic photonic crystal modulated by a nematic liquid crystal. Phys. B 388, 124-129 (2007)

17. C.Y. Liu, N.W. Zhang, H.W. Wang, L.W. Chen, Efficient tunable negative refraction photonic crystal achieved by an elliptic rod lattice with a nematic liquid crystal. Phys. B 404, 4060-4070 (2009)

18. G. Sun, A.G. Kirk, Analyses of negative refraction in the partial bandgap of photonic crystals. Opt. Express 16(6), 4330-4336 (2008) 
19. L. Shi, H. Yin, X. Zhu, X. Liu, J. Zi, Direct observation of isofrequency contour of surface modes in defective photonic crystals in real space. Appl. Phys. Lett. 97(251111), 1-3 (2010)

20. Y.Y. Wang, L.W. Chen, Tunable negative refraction photonic crystals achieved by liquid crystals. Opt. Express 14, 10580-10587 (2006)

21. G.V. Eleftheriades, K.G. Balmain, Negative-Refraction Metamaterials: Fundamental Principles and Applications (Wiley, Hoboken, New Jersey, 2005)

22. A. Taflove, Advances in Computational Electrodynamics: The Finite-Difference Time-Domain Method: A survey of The FiniteDifference Time-Domain Literature (Artech House, London, United Kingdom, 1998), pp. 1-30
23. C. Kittel, Introduction to Solid State Physics (Wiley, New York, USA, 2005)

24. E.H. Lock, The properties of isofrequency dependences and the laws of geometrical optics. Phys. Usp. 51(4), 375-393 (2008)

25. A.S. Spitsyn, G.F. Glinskii, Properties of waveguide modes in a photon crystal based on slotted silicon with a defect. Semiconductors 42(10), 1237-1243 (2008)

26. A. Yariv, P. Yeh, Photonics: Optical Electronics in Modern Communications (Oxford University Press, Oxford, USA, 2007)

27. A.V. Kildishev, V.M. Shalaev, Transformation optics and metamaterials. Phys. Usp. 54(1), 53-63 (2011) 